\title{
SPONTANEOUS RUPTURE OF A PREVIOUSLY SCARED UTERUS. A CASE REPORT AND AN OVERVIEW OF RISK FACTORS IN YEMEN REPUBLIC
}

\author{
Ishraq Dhaifalah
}

Department of Obstetric and Gynecology, Althawra Hospital, Sana'a University, Sana'a, Yemen Republic Department of Human Genetic and Fetal Medicine, University Hospital, I. P. Pavlova 6, 775 20 Olomouc, Czech Republic

Received: October 10, 2001

Key words: Spontaneous rupture of uterus / Previous scarring / Risk factors

Maternal mortality is a significant public health problem. In Yemen it is attributable to socioeconomic, reproductive, health-status and health-services factors, as well as to medical causes.

Direct obstetrical causes account for $61 \%$ of maternal deaths in Yemen $75 \%$ after delivery ${ }^{1}$. Hemorrhage has been found as the second cause of maternal death in Yemen, accounting for $23.68 \%$ of all causes, while ruptured uterus accounts for $14.19 \%$ of maternal deaths in particular. The high percentage of ruptured uterus as a cause of maternal mortality prompted me to report the case below hoping it would shed light on the influence of factors, which can lead to this serious situations in Yemen.

\section{INTRODUCTION}

Rupture of the uterus is a catastrophic event to both mother and fetus. And in a previously scarred uterus, especially after cesarean section, the risk of rupture is greatly increased. This condition is even more dangerous when dehiscence develops at a place where medical facilities are not available ${ }^{3}$. Many other factors also play an important role in increasing the incidence of uterine rupture or dehiscence of a previous cesarean section scar, for example:

- skill and experience of the surgeon

- state of patient at the time of operation

- multiparity

- post-operative complications that may occur, as infections, which is a very important factor for the progress of the next pregnancy and delivery

- and the type of cesarean section done.

It is well known that classical cesarean section is obsolete in modern obstetric and only indicated in cases of malformation of lower segment which may occur in transverse $\operatorname{lie}^{2}$. So when, the previous cesarean section was of the classical type, added to uterine anomalies, the subsequent rupture is much more probable. Thus subseptet uterus will narrow the uterine cavity and a classical cesarean section will increase the incidence of rupture. Such an accumulation of risk factors may lead to rupture at an early stage of gestation ${ }^{4}$.

Here I report a case of rupture of the uterus having several of the above mentioned risk factors.

\section{CASE REPORT}

24 year-old- women G 2, P $1+0$ was admitted at 30-week gestation owing to severe, acute lower abdominal pain like a stab that awakened her from sleep followed by an aggressive fetal movement at 4. a. m at home. Since then, pain was continuous and accompanied by mild vaginal bleeding with watery discharge. Four years previously she had a cesarean section, owing to a transverse lie with an outcome of a dead fetus. No other significant surgical history. Medical history included tuberculosis diagnosed three months earlier, for which the patient was on treatment till admission. Family history revealed tuberculosis of her sister-in-law. She was currently chewing Qat and stopped smoking seven months ago after being a smoker for five years. The patient was told not to conceive for four years after the operation and was still on contraceptive pills when she got pregnant. On admission the patient was uncomfortable with little movement, blood pressure 120/70, pulse $112 / \mathrm{min}$, temperature $37^{\circ} \mathrm{C}$ and respiratory rate $16 / \mathrm{min}$.

On examination her abdomen was rigid and tender fetal parts were easily felt with no cardiac activity. Vaginal examination showed $1.5 \mathrm{~cm}$ long dilated cervix, posterior, thick with blood stained liquor amnii. Rupture of the uterus complicating pregnancy was diagnosed, and the patient was immediately transferred to the operating theater. Under general anesthesia was performed sub-umbilical para-median incision laparotomy and a dead female fetus with placenta were removed from the abdominal cavity. A sub-septet uterus with dehiscence of a previous classical cesarean section that extended with a rupture down to the cervix was found to be the site of rupture. Resection of the septus and repair of 
the rupture was performed. Oozing from previous adhesions was controlled and lysis of some was done. The abdomen was closed in layers. Blood loss was about $500 \mathrm{ml}$ and urine was clear throughout operation. Operating time was about $80 \mathrm{~min}$ and the patient recovered in $15 \mathrm{~min}$, no blood transfusion. Post operatively the patient was covered with a broad-spectrum antibiotic. The first day postoperatively the urinary catheter was removed and for the six days she progressed well and was discharged on the seventh day postoperatively with a pulse $85 / \mathrm{min}$. temp $36.5^{\circ} \mathrm{C}$ and blood pressure $120 / 80$, blood hemoglobin $10.5 \mathrm{gr} . / \mathrm{dl}$. The patient was advised to come back for tubal ligation later.

\section{COMMENT}

Our patient was lucky enough to survive as she had many factors exposing her to the risk of rupture uterus. She had a previous classical cesarean section, sub-septet uterus and she is not educated, which in my opinion played a very important role in this patient and in other patients as well. If this patient had been educated she may have sought for early antenatal care, for early diagnosis and management, and this might have prevented the losses of both pregnancies. She would also have been able to give us a better history of her previous cesarean, and generally, taken better care of herself and her baby.

The misfortune for this patient was in having both pregnancies ended by complicated operations and intrauterine fetal death: the first one was classical cesarean section, and the second one was surgery for ruptured uterus and late diagnosis of sub-septet uterus. Thus the patient lost chances for any further safe pregnancy. It was very difficult to take the decision of tubal ligation during the operation. Our patient was young, had no children, and there was no previous agreement because of unknown history of the type of cesarean operation and of the sub-septet uterus. In addition to the culture of this woman where, fertility is of the utmost importance in the life of a married women ${ }^{1,6}$.

Hemorrhage in general is still the cause of maternal death in cases of ruptured uterus ${ }^{5}$. The percentage of ruptured uterus is much lower today especially in developed countries. But in our country there is still a high percentage with an average of 60 cases per year in a hospital with about 8000 deliveries per year. (Althawra, one of the main hospitals in Sana'a, Yemen). The causes of ruptured uterus in Yemen can not be defined on medical bases alone. There are causes behind causes, which are multi-layered, for the majority of cases of ruptured uterus. A woman in Yemen will not have uterine rupture because of medical causes only. She will have it because she does not receive adequate and timely treatment, she is a grand multi-para in addition of being in poor general health, did not know about contraceptives to prevent pregnancy and had no access to family planing services. Generally it is because she is illiterate, poor and lives in a rural area. Thus it is a failure in a chain of events which are collectively responsible for these cases.

\section{REFERENCES}

1. Abdulghani, N. (1993) Risk factors for Maternal Mortality among women using Hospitals in North Yemen. A Thesis presented for the degree of PhD. D. in the Faculty of Medicine University of London.

2. Chamberlain, G. (1999) Obstetric by ten teachers. Sixteenth edition: Great Britain, 308.

3. Leung, A. S., Leung, E. K., Paul, R. H. (1993) Uterine rupture after previous caesarean delivery: maternal and fetal consequences. Am. J. Obstet. Gynecol. 169, 945-950.

4. Nouria, M., Salama, A. et al. (1998) Pregnancy in the malformed uterus. Study of 366 pregnancies. Tunis Med. 76, 376-379.

5. Selo-Ojeme, D. O., Okonofua (1997) Risk factors for primary postpartum hemorrhage. A case control study. Arch. Gynecol. 259, $179-87$.

6. UNICEF (1992) The Situation of Children and Women in Rep. of Yemen, Sana'a. 